\title{
Commentary
}

Amos Grünebaum*, Laurence B. McCullough, Eran Bornstein, Risa Klein, Joachim W. Dudenhausen and Frank A. Chervenak

\section{Professionally responsible counseling about birth location during the COVID-19 pandemic}

https://doi.org/10.1515/jpm-2020-0183

Received April 25, 2020; accepted May 6, 2020; previously published online May 13, 2020

Abstract: If the worries about the coronavirus disease 2019 (COVID-19) pandemic are not already enough, some pregnant women have been questioning whether the hospital is a safe or safe enough place to deliver their babies and therefore whether they should deliver out-of-hospital during the pandemic. In the United States, planned out-ofhospital births are associated with significantly increased risks of neonatal morbidity and death. In addition, there are obstetric emergencies during out-of-hospital births that can lead to adverse outcomes, partly because of the delay in transporting the woman to the hospital. In other countries with well-integrated obstetric services and welltrained midwives, the differences in outcomes of planned hospital birth and planned home birth are smaller. Women are empowered to make informed decisions when the obstetrician makes ethically justified recommendations, which is known as directive counseling. Recommendations are ethically justified when the outcomes of one form of management is clinically superior to another. The outcomes of morbidity and mortality and of infection control and prevention of planned hospital birth are clinically superior to those of out-of-hospital birth. The obstetrician therefore should recommend planned hospital birth and recommend against planned out-of-hospital birth during the COVID-19 pandemic. The COVID-19 pandemic has

*Corresponding author: Amos Grünebaum, MD, Department of Obstetrics and Gynecology, Lenox Hill Hospital, 100 East $77^{\text {th }}$ St., New York, NY 10075, USA; and Department of Obstetrics and Gynecology, Zucker School of Medicine at Hofstra/Northwell, Hempstead, New York, NY, USA, E-mail: amos.grunebaum@gmail.com Laurence B. McCullough, Eran Bornstein, Risa Klein and Frank A. Chervenak: Department of Obstetrics and Gynecology, Zucker School of Medicine at Hofstra/Northwell, Hempstead, New York, NY, USA Joachim W. Dudenhausen: Department of Obstetrics and Gynecology, Zucker School of Medicine at Hofstra/Northwell, Hempstead, New York, NY, USA; Lenox Hill Hospital, Zucker School of Medicine at Hofstra/Northwell, New York, NY, USA; and Faculty of Health Sciences Brandenburg, Potsdam, Germany increased stress levels for all patients and even more so for pregnant patients and their families. The response in this difficult time should be to mitigate this stress and empower women to make informed decisions by routinely providing counseling that is evidence-based and directive.

Keywords: birth center; birth location; counseling; COVID-19 pandemic; home birth; professional ethics in obstetrics; professional virtue of integrity; public health emergency.

\section{Introduction}

If the worries about the coronavirus disease 2019 (COVID-19) pandemic are not already enough, some pregnant women have been questioning whether the hospital is a safe or safe enough place to deliver their babies and therefore whether they should deliver out-of-hospital during the pandemic. In several countries, this has become a lively topic for discussion in newspapers, journals, and social media [1-7]. In the United Kingdom, the National Health Service has suspended home births. In response, some pregnant women have turned to "freebirths" [8, 9]. In Germany, major physician organizations advise against home birth [10]. The purpose of this paper is to provide guidance for obstetricians to provide professionally responsible counseling about the impact of the COVID-19 pandemic on the safety of hospital birth vs. out-of-hospital birth. We first review the evidence base and then identify the implications of this evidence for professional ethics in obstetrics.

\section{Evidence base}

\section{Outcomes of out-of-hospital birth compared to planned hospital birth}

In the United States, planned out-of-hospital births are associated with significantly increased risks of neonatal morbidity and death [11-17]. In addition, there are 
obstetric emergencies during out-of-hospital births that can lead to adverse outcomes, partly because of the delay in transporting the woman to the hospital [18]. In other countries with well-integrated obstetric services and welltrained midwives, the differences in outcomes of planned hospital birth and planned home birth are smaller [19].

Current data about outcomes in the United States can be summarized as follows:

- Planned out-of-hospital births are known to increase neonatal deaths and injuries many-fold, especially for many women who are at increased risks [11-17].

- Even out-of-hospital birth centers have been associated with higher neonatal mortality risks when compared to hospital births [11]. While risks at out-of-hospital birth centers in the US are slightly lower than home births, the risks at out-of-hospital birth centers are still significantly higher than planned hospital births [11].

- Home births do not necessarily reduce the risk of complications; however, they increase the risks that complications will lead to brain injury for the baby or even death [18].

- There are significant risks when transporting patients to the hospital and the time involved is usually too long to prevent adverse outcomes [18].

- Hospital staff are well trained and better equipped to deal with emergencies than out-of-hospital providers. There are also more staff available than the 1-2 providers at home.

- At out-of-hospital births, there is no immediate access to blood transfusions, operating rooms for emergency cesarean deliveries, and either no access or not enough people who can perform advanced resuscitation and intubation of a baby who is born not breathing.

- Most if not all of known COVID-19-infected pregnant women have been infected prior to hospital admissions through community exposure or family members and friends.

\section{Infection control and prevention}

One theoretical reason to consider an out-of-hospital birth is that giving birth at an out-of-hospital location might reduce the risk of COVID-19 infection for both pregnant women and their babies. There are no data to support this hypothesis. However, with effective infection control and prevention in the hospital such as regular disinfections, separating known infected patients, and wearing personal protective equipment (PPE), there are negligible risks of patients becoming infected, and presently there is no documented increase in risk of infection. The only study available on the risk of neonatal infection in home vs. hospital births showed that there is an increased risk of infection-related mortality from planned home birth for newborns [11]. Labor and delivery departments in hospitals have implemented successful methods of infection control during the COVID pandemic which include, screening of patients and employees, COVID testing of all patients, strict visitation policies, appropriate use of PPE, isolation of affected or suspected patients, and appropriate disinfection and cleanliness methods.

In addition, midwives who do not have access to PPE or testing, or even refuse to wear masks [4], are likely to increase the risk of transmission. An unknown potential increased risk may result from family members who do not use PPE at planned home birth.

Current data about infection control and prevention in the United States can be summarized as follows:

- Out-of-hospital births do not protect from infections. Quite the opposite. Studies have shown babies dying at midwife-attended planned out-of-hospital births are significantly more likely to die from infection than hospital births [17].

- Hospitals are better able to protect patients from becoming infected by using regular disinfection, masks, face shields, and gowns.

\section{Counseling women about planned home birth during the COVID-19 pandemic}

\section{The information to be conveyed}

In professional ethics in obstetrics, the informed consent process serves to empower women to make informed decisions about intrapartum management [20, 21]. The obstetrician does so by providing women with current evidence about the relative outcomes of planned hospital birth compared to planned home birth and the risk of infection in both locations. Obstetricians can use the aforementioned summary points as a checklist to ensure the quality of this communication.

\section{Two take-home lessons for women}

Women will become further empowered to make informed decisions about birth location when the obstetrician emphasizes two take-home lessons. First, the perinatal outcomes of planned home birth are worse than those of planned hospital births in countries such as the United States, which does not have integrated 
obstetric services and which permits a wide variety of midwifery training and certification. For example, while all midwives in hospitals are certified-nurse midwives, most out-of-hospital midwives are not certified-nurse midwives. Second, the risk of infection from planned home birth is likely higher than that from planned hospital birth.

\section{The role for directive counseling}

Women are empowered to make informed decisions when the obstetrician makes ethically justified recommendations, which is known as directive counseling [20, 21]. Recommendations are ethically justified when the outcomes of one form of management is clinically superior to another. As demonstrated earlier, the outcomes of morbidity and mortality and of infection control and prevention of planned hospital birth are clinically superior to those of out-of-hospital birth. The obstetrician therefore should recommend planned hospital birth and recommend against planned out-of-hospital birth during the COVID-19 pandemic.

\section{Conclusion}

The COVID-19 pandemic has increased stress levels for all patients and even more so for pregnant patients and their families. The response in this difficult time should be to mitigate this stress and empower women to make informed decisions by routinely providing counseling that is evidence-based and directive.

Research funding: None declared.

Author contributions: All authors have accepted responsibility for the entire content of this manuscript and approved its submission.

Competing interests: Authors state no conflict of interest.

\section{References}

1. Accessed 4/23/2020: https://www1.wdr.de/nachrichten/ ruhrgebiet/corona-hausgeburten-nachfrage-gross-100.html.

2. Accessed 4/23/2020: https://theconversation.com/duringcoronavirus-hospital-surge-a-midwife-recommends-homebirth-133866.
3. Accessed 4/23/2020: https://www.nytimes.com/2020/03/30/ parenting/home-birth-coronavirus-hospital.html.

4. Accessed 4/23/2020: https://www.nytimes.com/2020/04/21/ nyregion/coronavirus-home-births.html.

5. Accessed 4/23/2020: https://www.washingtonpost.com/dcmd-va/2020/03/20/pregnant-women-worried-about-hospitalsamid-coronavirus-are-turning-home-births-an-alternative/.

6. Accessed 4/23/2020: https://www.businessinsider.com/ pregnant-people-last-minute-home-birth-requests-midwivesadvice-2020-3.

7. Accessed 4/23/2020: https://www.abc.net.au/news/202004-12/coronavirus-fears-drive-increase-in-homebirth-interest $/ 12138386$.

8. Accessed 4/23/2020: https://www.thesun.co.uk/news/11286252/ nhs-suspends-home-births-coronavirus-mums-babies-hospital/.

9. Accessed 4/23/2020: https://www.theguardian.com/lifeandstyle/2020/apr/05/expectant-mothers-turn-to-freebirthingafter-home-births-cancelled.

10. Accessed 4/23/2020: https://www.aerzteblatt.de/nachrichten/111827/COVID-19-Veto-zur-Hausgeburt.

11. Grünebaum A, McCullough LB, Sapra KJ, Brent RL, Levene MI, Arabin $B$, et al. Early and total neonatal mortality in relation to birth setting in the United States, 2006-2009. Am J Obstet Gynecol 2014;211:390.e1-7.

12. Grünebaum A, McCullough LB, Sapra KJ, Brent RL, Levene MI, Arabin B, et al. Apgar score of 0 at 5 minutes and neonatal seizures or serious neurologic dysfunction in relation to birth setting. Am J Obstet Gynecol 2013;209:323.e1-6.

13. Wax JR, Lucas FL, Lamont M, Cartin A, Blackstone J. Maternal and newborn outcomes in planned home birth vs planned hospital births: a metaanalysis. Am J Obstet Gynecol 2010;203:243.e1-8.

14. Grünebaum A, McCullough LB, Brent RL, Arabin B, Levene MI, Chervenak FA. Perinatal risks of planned home births in the United States. Am J Obstet Gynecol 2015;212:350.e1-6.

15. Grünebaum A, McCullough LB, Sapra KJ, Arabin B, Chervenak FA. Planned home births: the need for additional contraindications. Am J Obstet Gynecol 2017;216:401.

16. Grünebaum A, McCullough LB, Arabin B, Chervenak FA. Critical appraisal of the proposed defenses for planned home birth. Am J Obstet Gynecol 2019;205:1-5.

17. Grünebaum A, McCullough LB, Arabin B, Dudenhausen J, Orosz $B$, Chervenak FA. Underlying causes of neonatal deaths in term singleton pregnancies: home births versus hospital births in the United States. J Perinat Med 2017;45:349-57.

18. Grünebaum A, McCullough LB, Chervenak FA, Dudenhausen JW. Sudden severe fetal compromise at a planned home birth - a case of umbilical cord prolapse. Case Rep Perinat Med 2019;20190026. DOI: 10.1515/crpm-2019-0026.

19. Gesellschaft für Qualität in der außerklinischen Geburtshilfe e. V. Außerklinische Geburtshilfe in Deutschland - Qualitätsbericht, 2018. http://www.quag.de/quag/publikationen.htm.

20. Chervenak FA, McCullough LB. The unlimited-rights model of obstetric ethics threatens professionalism. Br J Obstet Gynaecol 2017;124:1144-7.

21. McCullough LB, Coverdale JH, Chervenak FA. Professional ethics in obstetrics and gynecology. Cambridge and New York: Cambridge University Press; 2020. 